\title{
Profesionales de la economía de la salud: quiénes somos y dónde trabajamos
}

\section{Health Economists in Spain: who we are and where we work}

Núm. 6 (2016), pp. 9-27

Trapero Bertran, Marta*1

Oliva-Moreno, Juan*2

Recibido: noviembre, 2014 Aceptado: enero, 2016

JEL Clasif: A11, A2, I1, J2

DOI: $10.5944 /$ reppp.6.2016.13458

\footnotetext{
*1 Marta Trapero Bertran, Investigadora y profesora asociada de la Universidad Castilla La-Mancha y Universitat Pompeu Fabra. E-mail: marta.trapero-bertran@upf.edu. ORCID: http://orcid.org/oooo-0oo29233-1776

*2 Juan Oliva Moreno, Profesor titular e investigador de la Universidad Castilla La-Mancha. E-mail: juan.olivamoreno@uclm.es
} 


\title{
Resumen
}

El objetivo principal de este estudio es describir las características de los profesionales que trabajan en el campo de la economía y gestión de la salud en España. Este trabajo presenta los datos de una encuesta realizada en el año 2009 a profesionales con interés en la economía de la salud y gestión sanitaria relacionados con la Asociación de Economía de la Salud (AES). 285 personas respondieron a la encuesta. El trabajo presenta información sobre las características de estos profesionales, su formación académica, aspectos relacionados con su mercado de trabajo, incluyendo ingresos por categoría profesional y tipo de empleo, información sobre la división de su tiempo de trabajo entre diferentes actividades, el tipo de investigación que desarrollan y sus percepciones respecto a aspectos curriculares.

Palabras clave: economistas de la salud; mercado de trabajo; recursos humanos; formación; ingresos

\begin{abstract}
The main objective of this study is to describe the characteristics of professionals working in the field of Health Economics and health care management in Spain. The paper presents data from a 2009 survey of professionals with interest on Health Economics and health management in the framework of the Spanish Health Economics Association and its Congress. 285 people responded the survey. We present summary information of the characteristics of these professionals, their education, a description of their job market, including earnings by rank and occupation, the time division, the type of research they are focused on and their perceptions of curricular issues.
\end{abstract}

Key Words: health economists; job market; human resources; education; incomes 


\section{Introducción}

Desde hace más de una década la demanda de economistas de la salud se ha incrementado sustancialmente en Europa. Ello se debe al creciente interés en la aplicación de medidas de eficiencia en los sistemas de salud y en el uso de la evaluación económica como una herramienta esencial para guiar la toma de decisiones. La irrupción de la crisis económica y su repercusión en los sistemas de salud no han hecho sino reforzar esta tendencia. Sin embargo, parece que las estructuras y el número de profesionales dedicados a la investigación, análisis y gestión de recursos en economía de la salud no son aún suficientes en los nuevos estados miembros de la Unión Europea (Gulácsi, 2007).

La Asociación de Economía de la Salud (AES) se constituyó formalmente en 1985. Los objetivos de AES son, entre otros, «contribuir a la mejora de la salud y de los servicios de atención sanitaria para el conjunto de la población, a través de las aportaciones del conocimiento científico; impulsar el debate en torno a alternativas que persigan la eficiencia y equidad en los sistemas de salud, para que repercutan en el bienestar general de la sociedad y favorezcan la sostenibilidad del sistema; promover el buen gobierno de los sistemas de salud, potenciando el desarrollo efectivo de las políticas de salud y la rendición de cuentas a la sociedad; contribuir a generar una cultura de evaluación en el conjunto de los sistemas de salud, abarcando desde el nivel de las políticas a la gestión clínica; estimular el interés por la Economía de la Salud en los y las profesionales que trabajan en los ámbitos de la Salud, la Sanidad y la Economía» (AES, 2012).

Siguiendo esta filosofía, AES se constituye en un foro de encuentro y debate para profesionales procedentes de diferentes disciplinas (no solo economía, administración o gestión sino también otras áreas sociales como las ciencias de la salud.) con interés en la economía de la salud y la gestión sanitaria. Estos profesionales desempeñan su labor en distintos ámbitos de diferente naturaleza, pública y privada, ocupando puestos de variada responsabilidad. Como resultado de este marco multidisciplinario, la economía de la salud es un campo que favorece la creación de redes de contacto profesional, pero al mismo tiempo, es complejo describir y acotar dicho ámbito debido a la variedad de actividades y perfiles profesionales potencialmente contenidos. Sin duda, esta elevada complejidad es causa de la falta de una descripción de su mercado de trabajo, pese a la conciencia de las buenas oportunidades laborales que se desarrollan en el mismo (Oliva, Zozaya, 2006).

El objetivo principal de este estudio es describir las características y actividad profesional de las personas dedicadas a la economía y gestión de la salud en España. La información generada puede ser de utilidad en una estrategia de fomento de los recursos humanos destinados a un ámbito como el señalado, clave para los procesos de evaluación de políticas, programas e intervenciones en salud y bienestar. Para ello, en primer lugar, se mostrará las características de las personas que trabajan en esta disciplina; en segundo lugar, se describirá la formación oficial adquirida por estos profesionales; en tercer lugar, analizaremos aspectos relacionados con el mercado de trabajo de la economía de la salud, especialmente desde la óptica de la oferta; en cuarto, se profundizará en aspectos de división del tiempo de trabajo entre diferentes tareas y deberes; en quinto lugar, se estudiará la actividad investigadora desarrollada por estos profesionales; y finalmente, también se analizará la opinión y percepción de los profesionales de este campo sobre aspectos curriculares. 


\section{Datos y métodos}

Los datos empleados en este trabajo se recogieron en una encuesta de sección cruzada realizada a economistas, gestores, investigadores en servicios sanitarios y en resultados en salud, y otros profesionales con interés en la economía y gestión de la salud. Para acceder a ellos se contó con la colaboración de la Asociación de Economía de la Salud, quien permitió difundir la encuesta entre sus socios y entre las personas que asistieron a alguna de las últimas Jornadas de Economía de la Salud. La encuesta fue diseñada por los propios autores a partir de un estudio previo realizado en los EE.UU. en 2005 por Morrisey y Cawley (2008). Se realizó una prueba piloto previa a la difusión de la encuesta, en la cual varios profesionales, de distintos ámbitos y perfiles, reportaron información de utilidad, la cual ayudó a perfilar la encuesta e introducir varios cambios en la versión definitiva. El cuestionario se divide en seis áreas diferentes: (i) características de los economistas y profesionales de la gestión de la salud; (ii) formación; (iii) mercado de trabajo; (iv) división del tiempo de trabajo; (v) tipo de investigación desarrollada; y (vi) opinión y percepción de los profesionales en este campo. La encuesta se realizó on-line mediante el software Survey Monkey. Los datos fueron analizados usando los programas Stata vs. 11 y Microsoft Excel.

Se enviaron invitaciones a participar a todas las personas afiliadas a la AES o participantes en las últimas Jornadas de Economía de la Salud con constancia de su correo electrónico (771 personas). Adicionalmente, fueron enviados dos recordatorios dentro del período del estudio a los 771 participantes potenciales. La encuesta estuvo abierta desde el 13 de julio hasta el 3 de diciembre de 2009.

\section{Resultados}

Esta sección se subdivide en seis temas de acuerdo con el diseño de las áreas de estudio. La tasa de respuesta fue del $37 \%$ (285 de los 771 encuestados) de todas las personas afiliadas de las cuales se disponía de una dirección de correo electrónico válida.

\subsection{Características de los economistas y profesionales de la gestión de la salud}

El 65,3\% de las personas encuestadas eran varones. Un 50\% tenía una edad inferior a 47 años, siendo el más joven de 24 años y el mayor de 73 . La nacionalidad más frecuente fue la española (91\%), siendo el 6\% de los encuestados de países latinoamericanos, como Perú, Uruguay o Argentina.

La mayoría de las personas que respondieron a la encuesta se describieron como economistas de la salud (33,3\%) o profesionales de la salud que trabajan con frecuencia en economía de la salud (32\%). Solo el 5,35\% de los profesionales afirmaron que eran economistas que trabajaban ocasionalmente en temas de salud. 


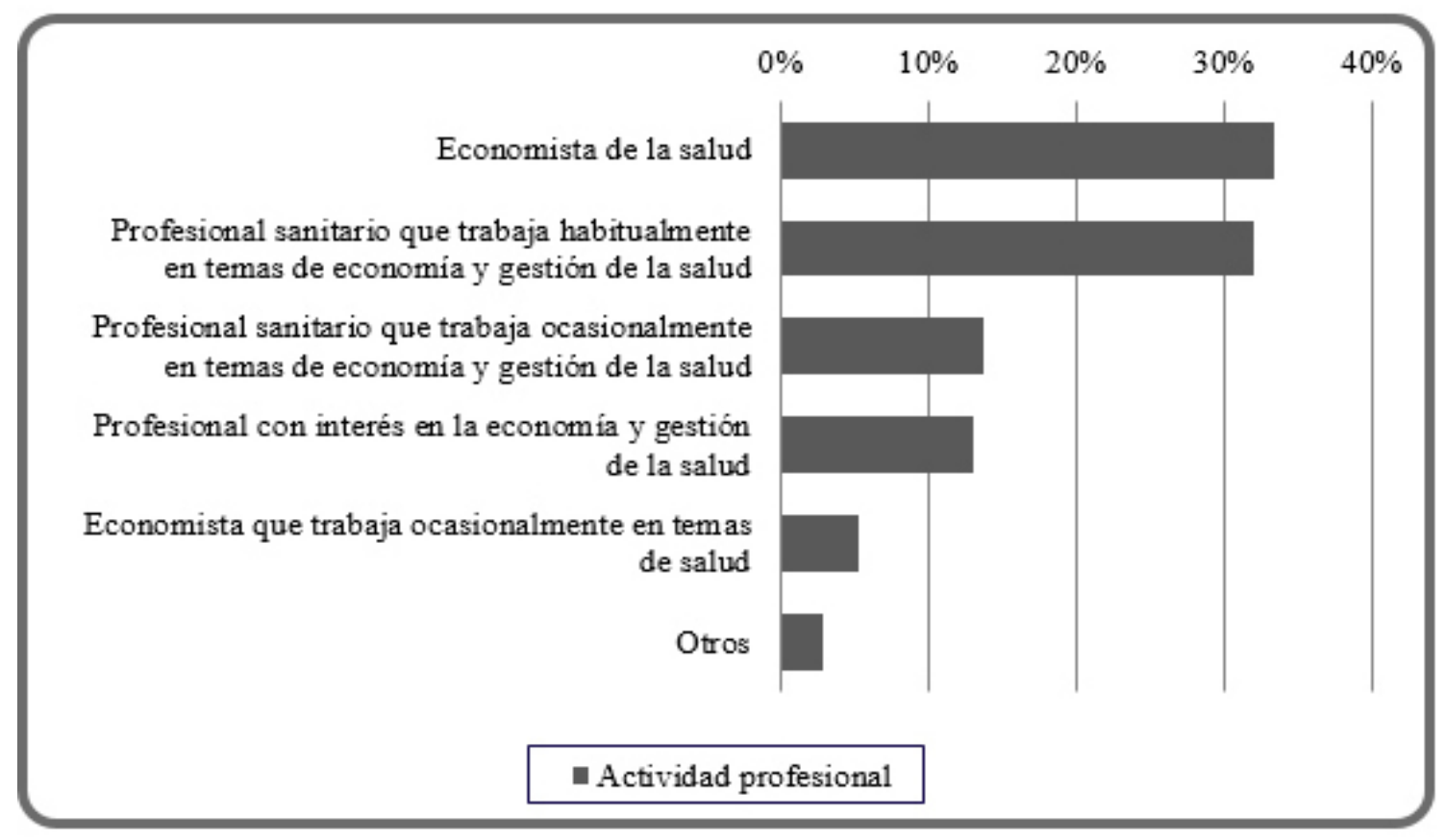

Gráfico I: Descripción de los puestos de trabajo (número de encuestados).

Fuente: elaboración propia.

En cuanto a la afiliación de las personas encuestadas, 270 de las 285 (94,7\%) estaban afiliadas a AES. Un 21,8\% estaban también afiliadas a otras asociaciones federadas en la Sociedad Española de Salud Pública y Administración Sanitaria (SESPAS) o hermanadas con AES (sociedades de economía de la salud del ámbito iberoamericano). Un 13,7\% de los encuestados pertenecían a otras asociaciones médicas como la Sociedad Española de Unidad Coronaria Aguda y Medicina Intensiva (SEMICYUC), Sociedad Española de Medicina de Familia y Comunidad (SEMFyC), la American Public Health Association (APHA), etc. Un 13,3\% de los encuestados respondieron estar también afiliados a la International Health Economics Association (IHEA), un 7,4\% a la International Society for Pharmacoeconomics and Outcomes Research (ISPOR); y un 2,5\% de los encuestados estaban vinculados a la Health Technology Assessment International (HTAi).

Durante los tres años previos a la encuesta, dos de cada tres encuestados habían asistido la Jornadas de Economía de la Salud organizadas por AES, uno de cada cuatro al Congreso de SESPAS y uno de cada cinco a la Conferencia Europea de Economía de la Salud (European Conference on Health Economics, ECHE) o al International Health Economics Association Congress (IHEA). El 14,7\% de los encuestados no habían asistido a ningún congreso. El 22,1\% de las personas encuestadas asistió a otras conferencias como el Congreso Nacional de Hospitales españoles, la Conferencia de la Asociación Española para la Calidad de Atención, la Conferencia de la Asociación Española de Epidemiología y otras conferencias o congresos nacionales e internacionales. 


\subsection{Formación}

Todas las personas encuestadas habían completado un grado de educación superior. El nivel educativo más alto obtenido fue el de doctorado, con el 33,6\% de las personas encuestadas. El 44,2\% poseía un título de máster y un 6,4\% de los encuestados indicó otro tipo de formación de postgrado. De las personas que habían realizado un máster o título de postgrado, la especialidad más común había sido economía de la salud (57\%) y la dirección y gestión de servicios sanitarios (46,1\%), ambos en España. De los profesionales que habían estudiado en Europa (16,4\%), la gran mayoría tenía formación en economía de la salud. Entre los encuestados de América Latina, la formación más común se dio en el campo de la dirección y gestión de servicios sanitarios. Aquellos que se habían formado en los Estados Unidos recibieron formación en economía de la salud y dirección y gestión de servicios sanitarios.

El 50\% de las personas encuestadas concluyeron sus estudios de postgrado entre 1973 y 2002, mientras el 50\% restante lo terminó entre 2003 y 2009. Las personas encuestadas asistieron a programas en diferentes universidades e instituciones de todo el país, si bien las instituciones citadas con mayor frecuencia fueron la Universidad de Barcelona y la Universidad Pompeu Fabra. El 26,3\% de los participantes recibieron una beca para realizar sus estudios de postgrado. De las entidades nacionales que dotaron dichas becas, los más citados fueron la administración central (Ministerio de Educación y Ministerio de Sanidad) y los gobiernos regionales (Cataluña, Galicia, Valencia, País Vasco); las universidades (Castilla La-Mancha, Pompeu Fabra, Politécnica de Valencia, Granada, Carlos III) y las fundaciones (Ramón Areces, Pedro Barrié de la Maza, Mapfre). Adicionalmente, entre las entidades que dotaron becas de formación en centros de estudios internacionales de las cuales se beneficiaron algunas personas encuestadas se mencionaron el British Council; la Universidad de California y el Baylor College of Medicine, en Houston.

De las personas que obtuvieron un título de doctorado (96/285), los programas seguidos fueron de Economía o de Ciencias de la Salud. De estas personas, un 7,3\% obtuvo su título de doctorado en Europa (fuera de España) y un 2\% en los EE.UU. El 50\% de los encuestados obtuvo su título entre los años 1978 y 1996, y el resto entre 1997 y 2009. Las personas encuestadas obtuvieron su título de doctorado en universidades nacionales como la Universidad de Barcelona, la Universidad Complutense, la Universidad Autónoma de Barcelona, la Universidad Autónoma de Madrid, la Universidad Granada o la Universidad de Málaga, mientras que las universidades internacionales citadas fueron la NorthWest University, la University of York y la London School of Economics. Solo el 19,8\% de los encuestados con el título de doctor se beneficiaron de una beca durante sus estudios de postgrado. El Ministerio de Educación y la Comisión Europea fueron los entes financiadores más citados.

Un 15\% de los participantes (43/285) se encontraban estudiando a tiempo parcial en el momento de ser encuestados, la mayoría realizando estudios de máster $(23,2 \%)$ o de doctorado $(34,9 \%)$. 


\subsection{Mercado de trabajo}

Un 96,1\% (274/285) de las personas encuestadas respondió esta sección. La gran mayoría (90,5\%) trabajaba en España. El 4,7\% trabajaba en otros países de Europa y el 4,7\% en América Latina. Un 66,9\% de las personas encuestadas estaban empleadas en el sector público (26\% en los centros y servicios de provisión de servicios sanitarios, un 15,6\% en el ámbito académico un 13,7\% en los servicios centrales de los gobiernos autonómicos, un 4,1\% en agencias de evaluación o centros de investigación pública dependientes de la administración autonómica, un 3,7\% en el Ministerio o institución pública dependiente de la administración central, y el resto en agencias de evaluación o centros de investigación pública dependientes de la administración central o en centros o entidades públicas dependientes de la administración local). El 29\% trabajaban en el sector privado, un $3,3 \%$ eran trabajadores por cuenta propia y el resto trabaja en otras instituciones como organizaciones no gubernamentales (ONG).

\section{Empleador}

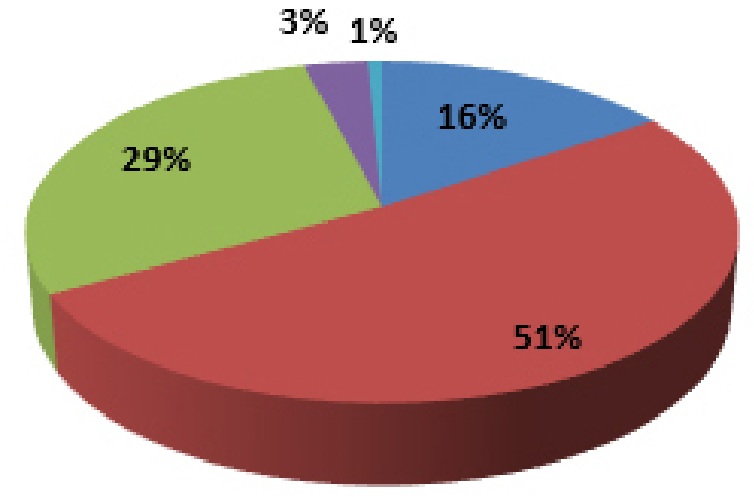

Público (Universidad)

Público (No universidad)

Privado

Trabaja por cuenta propia

Otras situaciones

Gráfico 2: Sector de trabajo de los encuestados (número de encuestados).

Fuente: elaboración propia

Un 92,0\% de las personas encuestadas (252/274) proporcionaron información sobre su nivel salarial. El rango salarial más frecuente de la actividad principal de los participantes fue de 30.001-45.000 $€$ anuales (año base: 2009) en el caso de personas empleadas en el sector público; 45.001-60.00o $€$ en el caso de los trabajadores por cuenta propia; y superior a $60.001 €$ para las personas empleadas en el sector privado. En general, considerando todos los sectores, un 26,69\% de las personas encuestadas se encontraban en el intervalo salarial de 30.001 a $45.000 €$; un 25,90\% en el intervalo de 45.001 a $60.000 €$; y un $34.66 \%$ percibían más de 60.001 $€$ anuales. De estos participantes, el 
90\% trabajaban en España, desempeñando su labor en el sector público un 63,75\%; un 25,10\% en el sector privado; y un 1,99\% eran trabajadores por cuenta propia. Además de su ocupación principal, los participantes declararon tener ingresos adicionales por otras actividades, a las cuales dedicaban, en promedio, un 9,3\% de su tiempo total de trabajo y obtenían un ingreso adicional de $4.886 €$, en media. La Tabla 1 muestra, sobre 237 casos válidos, el salario bruto total anual promedio según la entidad contratante (pública o privada).

Tabla I. Salario bruto anual según sector de ocupación (€ 2009).

\begin{tabular}{|l|c|c|}
\hline & N=237 & $\begin{array}{c}\text { Salario bruto anual } \\
\text { total } \\
\text { Media (DE) }\end{array}$ \\
\hline $\begin{array}{l}\text { 1. Empleados públicos } \\
\text { universidad }\end{array}$ & 163 & $55.017(24.760)$ \\
\hline $\begin{array}{l}\text { 1.1. Universidad } \\
\text { 2. Empleados privados }\end{array}$ & 39 & $48.159(22.275)$ \\
\hline $\begin{array}{l}\text { 2.1. Empresa privada } \\
\text { 2.2. Fundaciones, ONG, } \\
\text { otros }\end{array}$ & 37 & $77.760(54.111)$ \\
\hline $\begin{array}{l}\text { 3. Empleado por cuenta } \\
\text { propia }\end{array}$ & 30 & 82.850 (5.599) \\
\hline
\end{tabular}

\section{Fuente: elaboración propia}

Para ubicar los rangos salariales declarados en el contexto español (dado que más de 9 de cada 10 personas encuestadas desarrollaba su actividad profesional en España), cabe señalar que el salario medio por trabajador en España en el año 2010 ascendió a $22.790 €$ anuales, de acuerdo con el Instituto Nacional de Estadística (Encuesta de Estructura Salarial-EES). El salario promedio de los graduados, ingenieros superiores y doctores fue de $35.865 €$ (un 57,4\% más alto que el salario promedio nacional de acuerdo con el ESS). Por lo tanto, los salarios de los economistas de la salud españoles son significativamente más altos que los salarios medios españoles de población activa con niveles educativos similares.

Dentro de las personas encuestadas, los empleados del sector público trabajaban principalmente en la universidad y en centros sanitarios, de planificación-gestión o de investigación dependientes de los gobiernos regionales que prestan servicios de salud, mientras que los empleados en el sector privado trabajaban principalmente en consultorías y empresas del sector farmacéutico. La mayoría de los encuestados que trabajaban en una universidad eran catedráticos y profesores titulares, seguidos de otras figuras contractuales como ayudantes, ayudantes doctores y contratados doctores. Las áreas de conocimiento donde trabajaban estos profesores correspondían mayoritariamente al campo de la economía, la empresa y la salud pública. 
Otra información recabada se relaciona con la experiencia profesional y el tipo de contrato laboral. Los profesionales encuestados se habían mantenido en el mismo departamento, empresa o institución durante 9,3 años de media (DE: 7,9 años), con un rango que oscilaba entre un valor mínimo de o (recién llegado) y un valor máximo de 38 años. La gran mayoría de las personas encuestadas tenía un contrato permanente. Dos de cada tres de las personas encuestadas declaró haber recibido propuestas de empleo en los tres años anteriores al momento de realización de la encuesta.

Los encuestados que trabajaban en una universidad declararon que su actual universidad o departamento ofrecería un salario de, aproximadamente, de 27.465 $€$ anuales (DE: $12.407 €$ ) para un recién doctor contratado (basado en 49 respuestas), mientras que el sector privado podría ofrecer un salario de, aproximadamente, $34.954 €$ (DE: $10.795 €$ ) en promedio (basado en 68 respuestas). Para una persona con un título de máster recién adquirido, una universidad ofrecería un salario de alrededor de 27.474 $€$ (DE: 11.204 $€$ ) (basado en 41 respuestas), mientras que el sector privado ofrecería alrededor de $33.498 €$ (DE: $11.288 €$ ) (basado en 61 respuestas). Los estudios de doctorado en Economía eran el programa de doctorado más valorado por los encuestados en términos de contratación de personas para empresas o instituciones.

4 de las 274 personas encuestadas que respondieron a esta sección habían obtenido una beca para financiar los estudios de máster o programas de doctorado que cursaban en el momento de realizar la encuesta. El importe anual de la beca fue, en media, de 7.00o $€$ (DE: $8.981 €$ ). Estas personas dedicaban un 17,5\% (DE: 23,6) de su tiempo de trabajo y estudio a la realización de actividades adicionales para obtener ingresos extra. Los ingresos extra obtenidos eran en promedio de $2.875 €$ anuales (DE: $3.473 €$ ).

\subsection{División del tiempo de trabajo}

La tasa de respuesta en esta sección fue del 79,3\% (226/285). Las horas de trabajo semanales promedio declaradas fueron 47,5 (DE: 11.89 horas). El tiempo de estos profesionales se dividía de acuerdo a 6 actividades diferenciadas: (a) investigación (45\% del tiempo dedicado por los profesionales que desempeñan su actividad en la universidad, el $11 \%$ del tiempo dedicado por los profesionales que desempeñan su actividad en el sector público y el 10\% del tiempo dedicado por los profesionales que desempeñan su actividad en el sector privado); (b) análisis (6\% del tiempo ocupado en la universidad, el 19\% en el sector público y el $18 \%$ en el sector privado); (c) la enseñanza y la formación ( $35 \%$ del tiempo dedicado en la universidad; $9 \%$ en el sector público y el 10\% en el sector privado); (d) la gestión (10\% del tiempo en la universidad, el 37\% en el sector público y el $32 \%$ en el sector privado); (e) administración ( $3 \%$ del tiempo ocupado en la universidad, el $15 \%$ en el sector público, y el 9\% en el sector privado); (f) comercialización (o\% de tiempo en la universidad; $3 \%$ en el sector público y el 11\% en el sector privado); $\mathrm{y}$, (g) otras actividades (1\% del tiempo en la universidad; $6 \%$ en el sector público y el 10\% en el sector privado).

Solo el 20\% de los encuestados habían participado al menos una vez como miembros de un tribunal de tesis doctoral. En los 12 meses anteriores, 27 encuestados habían examinado, en media, 1,63 tesis doctorales, mientras que el número medio de tesis doctorales examinadas durante toda su carrera fue de alrededor de 9. De todas las tesis doctorales examinadas, alrededor de 4 (DE: 4,20; min. 1, máx. 20) se llevaron a cabo en el campo de la economía y gestión de la salud. 
Un 24,4\% de las personas encuestadas estaban dirigiendo o habían dirigido en algún momento proyectos de investigación en economía y gestión de la salud. Durante los 12 meses previos a la encuesta, el $73 \%$ de los encuestados habían participado, en calidad de docentes, en masters y cursos de postgrado de corta duración. De las personas que respondieron a esta sección, 67 habían dirigido o tutorizado tesinas de fin de máster; 36 habían dirigido o tutorizado trabajos en cursos pre-doctorales; y 30 habían dirigido tesis doctorales.

\subsection{Tipo de investigación}

La tasa de respuesta correspondiente a esta sección fue del 79,3\% (226 de cada 285 participantes). Las personas que respondieron afirmaron que invertían una media del 47,1\% de su tiempo de investigación en temas relacionados con la economía y gestión de la salud. Las áreas más comunes de investigación dentro de este campo fueron la evaluación económica, la investigación de resultados y la gestión clínica y sanitaria. El 70\% de las personas que respondieron a esta sección declararon haber publicado en revistas científicas.

Dentro de este grupo, el número de publicaciones en revistas internacionales durante su carrera varia bastante según el perfil del profesional, comprendiendo valores como 11,5 artículos de promedio (DE:20,3) para los profesionales sanitarios que trabajan ocasionalmente en temas de economía y gestión de la salud, como 1,3 artículos de promedio (DE: 2,0) para aquellos profesionales con interés en la economía y gestión de la salud, predominando las publicaciones en revistas de servicios sanitarios y análisis de políticas. La cantidad promedio más elevada de artículos publicados en revistas nacionales durante toda su carrera fue de 17,1 (DE: 50,1, $\mathrm{n}=11$ ) para los profesionales con interés en la economía y gestión de la salud, predominando las revistas del ámbito clínico-biomédico. El número medio total de artículos publicados durante toda su carrera fue de 24 artículos por persona (8 en revistas internacionales y 16 en revistas españolas), si bien hay que considerar que los tamaños muestrales varían entre categorías. Cuando se preguntó a los profesionales sobre el número de artículos publicados en los cinco años previos, el número promedio de artículos publicados en revistas internacionales se reduce a 3,7 publicaciones, mientras que el número de artículos en revistas nacionales se reduce a 4,4. Por lo tanto, en los cinco años anteriores a la encuesta, el número promedio de artículos publicados, ya sea en revistas nacionales o internacionales, fue de 8 artículos por persona. En general, incluyendo a los encuestados que no habían editado o publicado ningún libro, los participantes declararon ser autores o editores de una media de 1,8 libros por persona durante su carrera, cayendo este número a 1 libro por persona en los cinco años anteriores a la encuesta. La media de capítulos publicados por cada profesional fue de 4,2 reduciéndose a 2 en los cinco años previos a la encuesta. 
Tabla 2. Distribución de los resultados de investigación según el perfil de profesional $(n=\mid 38)$.

\begin{tabular}{|c|c|c|c|c|c|c|c|c|}
\hline \multirow[t]{2}{*}{ Perfil del profesional } & \multicolumn{2}{|c|}{$\begin{array}{l}\text { Artículos publicados } \\
\text { en revistas } \\
\text { internacionales } \\
\text { Media (DE) }\end{array}$} & \multicolumn{2}{|c|}{$\begin{array}{l}\text { Artículos publicados } \\
\text { en revistas nacionales }\end{array}$} & \multicolumn{2}{|c|}{ Libros publicados } & \multicolumn{2}{|c|}{$\begin{array}{l}\text { Capítulos de libros } \\
\text { publicados }\end{array}$} \\
\hline & $\begin{array}{l}\text { Toda la } \\
\text { carrera }\end{array}$ & $\begin{array}{l}\text { Últimos } 5 \\
\text { años }\end{array}$ & $\begin{array}{l}\text { Toda la } \\
\text { carrera }\end{array}$ & $\begin{array}{l}\text { Últimos } \\
5 \text { años }\end{array}$ & $\begin{array}{l}\text { Toda la } \\
\text { carrera }\end{array}$ & $\begin{array}{l}\text { Últimos } 5 \\
\text { años }\end{array}$ & $\begin{array}{l}\text { Toda la } \\
\text { carrera }\end{array}$ & $\begin{array}{l}\text { Últimos } 5 \\
\text { años }\end{array}$ \\
\hline Economista de la salud $(n=57)$ & $5,3(6,7)$ & $3,3(4,1)$ & $8,8(13,5)$ & $4,4(6,4)$ & $1,4(2.0)$ & $0,6(1.1)$ & $4,5(6,1)$ & $2,1(2, .7)$ \\
\hline $\begin{array}{l}\text { Economista que trabaja ocasionalmente } \\
\text { en temas de salud }(n=7)\end{array}$ & $8,4(6,6)$ & $3,4(2,4)$ & $6.3(5.6)$ & $2,1(3,1)$ & $1(1,4)$ & $0,4(0,5)$ & $2(1,6)$ & $1,4(1,4)$ \\
\hline $\begin{array}{l}\text { Profesional sanitario que trabaja } \\
\text { ocasionalmente en temas de economía y } \\
\text { gestión de la salud ( } n=18 \text { ) }\end{array}$ & $\begin{array}{c}11,5 \\
(20,3)\end{array}$ & $2,5(2,5)$ & $16,8(20,1)$ & $6,5(7,3)$ & $2,2(3)$ & $1(1,8)$ & $3,4(4,9)$ & $1,9(3)$ \\
\hline $\begin{array}{l}\text { Profesional de la salud que trabaja } \\
\text { habitualmente en temas de economía y } \\
\text { gestión de la salud ( } n=43 \text { ) }\end{array}$ & $4,3(7,9)$ & $1,5(2,6)$ & $11,6(21,6)$ & $4,8(7,7)$ & $0,9(1,7)$ & $0,5(1,2)$ & $3,5(6,5)$ & $1,5(3.0)$ \\
\hline $\begin{array}{l}\text { Profesional con interés en la economía y } \\
\text { gestión de la salud ( } n=11)\end{array}$ & $1,3(2,0)$ & $0,5(0,7)$ & $17,1(50,1)$ & $2,4(5,9)$ & $0,4(1,2)$ & $0,1(0,3)$ & $4,7(7,4)$ & $1,1(1,6)$ \\
\hline Otros $(n=2)$ & $15(0)$ & $11(5.6)$ & $37(32.5)$ & $6(1.4)$ & $5(2.8)$ & $3.5(2.1)$ & $7(5.6)$ & $4(2.8)$ \\
\hline
\end{tabular}

Fuente: elaboración propia. 
Tabla 3. Distribución de los resultados de investigación según sector $(n=\mid 35)$.

\begin{tabular}{|c|c|c|c|c|c|c|c|c|}
\hline \multirow[t]{2}{*}{ Sector } & \multicolumn{2}{|c|}{$\begin{array}{c}\text { Artículos publicados } \\
\text { en revistas } \\
\text { internacionales } \\
\text { Media (DE) }\end{array}$} & \multicolumn{2}{|c|}{$\begin{array}{l}\text { Artículos publicados en } \\
\text { revistas nacionales }\end{array}$} & \multicolumn{2}{|c|}{ Libros publicados } & \multicolumn{2}{|c|}{$\begin{array}{l}\text { Capítulos de libros } \\
\text { publicados }\end{array}$} \\
\hline & $\begin{array}{l}\text { Toda la } \\
\text { carrera }\end{array}$ & $\begin{array}{c}\text { Últimos } 5 \\
\text { años }\end{array}$ & $\begin{array}{l}\text { Toda la } \\
\text { carrera }\end{array}$ & $\begin{array}{c}\text { Últimos } 5 \\
\text { años }\end{array}$ & $\begin{array}{l}\text { Toda la } \\
\text { carrera }\end{array}$ & $\begin{array}{c}\text { Últimos } 5 \\
\text { años }\end{array}$ & $\begin{array}{l}\text { Toda la } \\
\text { carrera }\end{array}$ & $\begin{array}{c}\text { Últimos } 5 \\
\text { años }\end{array}$ \\
\hline Público ( $n=94)$ & $6,6(10,9)$ & $3,1(3,9)$ & $15(25,7)$ & $5,6(7,8)$ & $1,4(2,1)$ & $0,8(1,4)$ & $5,3(6,8)$ & $2,4(3,0)$ \\
\hline Universidad $(n=35)$ & $8,8(7,4)$ & $4,9(4,2)$ & $14,4(18,5)$ & $5,6(7,4)$ & $1,9(2,3)$ & $0,9(1,2)$ & $6,1(6,9)$ & $2,7(2,9)$ \\
\hline $\begin{array}{l}\text { Sector público (sin } \\
\text { incluir } \\
\text { Universidad) } \\
(\mathrm{n}=59)\end{array}$ & $5,2(12,4)$ & $2(3,2)$ & $15,3(29,3)$ & $5,6(8,0)$ & $1,1(2,0)$ & $0,7(1,5)$ & $4,8(6,8)$ & $2,3(3,1)$ \\
\hline $\begin{array}{l}\text { Sector privado } \\
\text { (incluyendo } \\
\text { trabajadores por } \\
\text { cuenta propia) } \\
(\mathrm{n}=41)\end{array}$ & $4,2(7,6)$ & $1,4(2,2)$ & $4,9(6,4)$ & $2,3(2,8)$ & $1,1(1,9)$ & $0,3(0,8)$ & $1,3(1,7)$ & $0,5(1,0)$ \\
\hline
\end{tabular}

Fuente: elaboración propia. 
Ante la pregunta de en qué revista habían sentido mayor satisfacción al ser aceptados sus trabajos o qué publicaciones les habían ayudado más en su proyección profesional, las respuestas difieren según el sector en el que los encuestados desarrollaban su actividad profesional. Así, en el campo académico y privado, la revista identificada como aquella donde produce mayor satisfacción publicar fue Health Economics, mientras que para el sector público (sin contar el sector académico) fue Gaceta Sanitaria. Entre las revistas más citadas también figuran Journal of Health Economics, European Journal of Health Economics y Medicina Clínica. Cuando se les preguntó sobre la revista donde aún no habían publicado pero les gustaría publicar en los próximos años, Journal of Health Economics ocupa de manera destacada la primera posición para los profesionales académicos y del sector privado, seguida de Health Economics, mientras que para el sector público (sin contar el sector académico) el British Medical Journal es la más valorada.

En relación con el proceso de revisión y evaluación en las revistas científicas editadas en España, el 38\% de los profesionales se sentían satisfechos aunque un elevado porcentaje (44\%) se mostraba indiferente a esta cuestión. En cambio, cuando se trata del proceso de revisión y evaluación en las revistas científicas editadas fuera de España, el 43\% de los profesionales se mostraban satisfechos mientras que, también en esta ocasión, un $45 \%$ se mostraba indiferente.

El 34\% de los encuestados declararon estar satisfechos con el proceso de evaluación de los programas nacionales de investigación. El 22\% está satisfecho con los procesos de evaluación de programas internacionales de investigación. Un 24\% de los profesionales se mostraban indiferentes a los procesos de evaluación de programas internacionales en comparación con los programas nacionales, seguramente porque hay menos investigadores que acceden a programas internacionales que nacionales y resulta más complicado que tengan una opinión formada. Un 40\% de los encuestados declaró estar satisfecho con los procesos de evaluación de los trabajos o resúmenes presentados a congresos nacionales e internacionales.

\subsection{Percepciones de los profesionales}

En este apartado se describe la percepción de los profesionales con interés en la economía y gestión de la salud sobre qué aspectos eran los más valorados en el currículum vitae (CV) en su medio de trabajo en el momento de realización de la encuesta y sobre el grado de acuerdo con algunos mensajes propuestos sobre su entorno laboral. La tasa de respuesta fue del 74,4\% (204 de 285).

Los profesionales con interés en economía y gestión de la salud valoraron los aspectos principales que tendrían en cuenta al analizar un CV, en una escala de o a 10, siendo o el peor y 10 la mejor puntuación de la misma. Un buen conocimiento de idiomas se considera relevante $(7,8$ sobre 10$)$ para un buen $\mathrm{CV}$. Con respecto a la importancia sobre dónde publicar su investigación, las revistas mejor puntuadas fueron aquellas relacionadas con economía de la salud (7,3 sobre 10) o con los servicios sanitarios (6,9 sobre 10). Sin embargo, revistas económicas más generales obtuvieron peor puntuación (5,9 sobre 10). La participación en proyectos de economía de la salud también se valoró como importante (7,6 sobre 10). La capacidad para colaborar con otras unidades y/o instituciones (7,9 sobre 10) $\mathrm{u}$ otras unidades en la misma empresa (8 sobre 10) fueron consideradas actividades de gran relevancia. Por su parte, la obtención de proyectos y contratos de investigación 
(7,5 sobre 10), la experiencia profesional previa en una posición similar $(8,1$ sobre 10$)$ y la capacidad de comunicarse de manera efectiva ante la audiencia $(8,3$ sobre 10$)$ se indicaron como los aspectos importantes en términos de la valoración de un CV.

Tabla 4. Aspectos valorables en el Curriculum Vitae según sector.

\begin{tabular}{|c|c|c|c|}
\hline Aspectos a considerar & Universidad & $\begin{array}{l}\text { Sector público } \\
\text { (sin incluir } \\
\text { universidad) }\end{array}$ & $\begin{array}{l}\text { Sector } \\
\text { Privado }\end{array}$ \\
\hline Conocimiento de idiomas & 7,6 & 7,8 & 8,1 \\
\hline Publicaciones en revistas económicas & 7,5 & 5,5 & 5,4 \\
\hline Publicaciones en revistas de Economía de la Salud & 8,4 & 6,9 & 6,9 \\
\hline $\begin{array}{l}\text { Publicaciones en revistas sobre Servicios } \\
\text { Sanitarios }\end{array}$ & 7,0 & 6,9 & 6,6 \\
\hline Publicaciones en revistas sobre Política Sanitaria & 7,0 & 6,5 & 6,1 \\
\hline Publicaciones en revistas biomédicas & 6,1 & 6,3 & 6,3 \\
\hline $\begin{array}{l}\text { Colaboración en proyectos de Economía de la } \\
\text { Salud }\end{array}$ & 8,2 & 7,4 & 7,3 \\
\hline $\begin{array}{l}\text { Capacidad de trabajo junto con otras unidades y } \\
\text { departamentos dentro de la misma institución }\end{array}$ & 7,9 & 7,9 & 8,2 \\
\hline $\begin{array}{l}\text { Capacidad de trabajo junto con otras instituciones } \\
\text { o compañías }\end{array}$ & 7,9 & 7,8 & 8,1 \\
\hline Obtención de becas o subvenciones & 8,5 & 7,3 & 7,0 \\
\hline $\begin{array}{l}\text { Colaboraciones para la gestión dentro de la misma } \\
\text { institución o compañía }\end{array}$ & 6,2 & 6,9 & 6,7 \\
\hline Experiencia profesional previa & 7,3 & 8,0 & 8,5 \\
\hline $\begin{array}{l}\text { Organización de congresos, asistencia a } \\
\text { conferencias, paneles de expertos, etc. }\end{array}$ & 7,3 & 6,8 & 6,9 \\
\hline Bien valorado en enseñanza & 7,6 & 7,1 & 6,4 \\
\hline Aptitudes de comunicación & 7,7 & 8,2 & 8,7 \\
\hline
\end{tabular}

Nota: Valores comprendidos entre 0 - 10 , siendo 10 el mejor valor. 
Los profesionales también dieron su opinión con respecto a diversos mensajes relacionados con su ambiente de trabajo principal. El 88\% de los profesionales coincidía en que la calidad de las publicaciones debería ser más importante que la cantidad para la promoción profesional. Además, el 30\% de los profesionales no creía que publicar como único autor debiera valorarse más que los trabajos con co-autoría. No obstante, un $55 \%$ estaba de acuerdo en que tiene más valor participar en una publicación de dos autores que en una de cinco o más. Más del 50\% de los encuestados coincidieron en que es más difícil promocionarse dentro de la disciplina de economía de la salud que otras disciplinas. El 63\% manifestó que su institución o empresa facilitaba la cooperación y el trabajo en equipo multidisciplinario, ya sea en la misma institución o con otras instituciones, empresas o departamentos. Alrededor del $46 \%$ admitió que había más de un 50\% de posibilidades de cambiar de trabajo durante los 3 años siguientes al momento de realización de la encuesta y alrededor de un 55\% afirmó que su entorno de trabajo facilitaba la conciliación de su vida profesional y personal.

\section{Discusión}

Los resultados obtenidos en este trabajo señalan que la economía de la salud en España es una actividad profesional multidisciplinar y no está en absoluto limitada al entorno académico de facultades de economía y empresa. El número de personas que se describieron a sí mismos como profesionales de la salud con interés en la economía de la salud es muy similar al de aquellos que se describieron como economistas de la salud.

La población encuestada fue relativamente joven y altamente cualificada en su formación reglada. Si comparamos nuestros resultados con los obtenidos en trabajos similares realizados en otros países (Morrisey y Cawley, 2008; Kaambwa y Frew, 2013), las características demográficas de los encuestados en España y en Estados Unidos son similares (porcentaje de hombres/mujeres y distribución por edades). Sin embargo, en la encuesta del Reino Unido, la distribución por sexo es marcadamente diferente ( $51 \%$ mujeres).

Los economistas de la salud trabajan en una amplia variedad de entornos, académicos y no académicos. La mayoría de las personas encuestadas $(66,9 \%)$ trabajaban para las Administraciones Públicas. Estos resultados son notablemente diferentes a los encontrados por Morrisey y Cawley (2008) y Kaambwa y Frew (2013), donde el 64\% de los economistas de la salud de Estados Unidos trabajaba en el entorno académico en: 2005 (65\% en Reino Unido), como empleados del gobierno (el gobierno federal casi exclusivamente) un $12 \%$ (en Reino Unido un 3\%), en organizaciones sin ánimo de lucro un 15\% (4\%) y un 9\% (20\%) en el sector privado. En EEUU (Morrisey y Cawley, 2008) el $100 \%$ de los encuestados eran economistas de formación, bien economistas de la salud (72\%) o economistas que trabajaban ocasionalmente en el campo de la salud (28\%). Por su parte, en España el 33\% se declararon economistas de la salud, el 32\% profesionales de la salud que trabajan con frecuencia en economía de la salud y, el $5 \%$ economistas que ocasionalmente trabajaban en economía de la salud. En EEUU, el 64\% de las personas encuestadas estaban empleadas en el ámbito académico (sector público), y en España el 16\%. En EEUU, el 93\% de los encuestados eran doctores, mientras en España este porcentaje era del $33,6 \%$. No obstante, estas diferencias en resultados deben ser interpretadas con cautela. De su observación no debe deducirse directamente que la actividad de la 
economía de la salud está mucho más centrada en las universidades en Estados Unidos o Reino Unido que en España. Una cuestión que debe ser considerada es el tipo de profesional asociado a las sociedades de economía de la salud en los tres países referidos. Toda vez que las personas encuestadas se localizaron a través de dichas asociaciones, si las metas y objetivos de las mismas difieren, también es razonable concluir que el tipo de perfil profesional no tiene por qué ser el mismo. En este sentido, uno de los objetivos de AES es atraer a profesionales de otras disciplinas interesados en la economía de la salud y no concentrar la participación en la asociación en un entorno exclusivamente académico.

Pasando a la parte de las retribuciones, los salarios anuales obtenidos por los profesionales en Estados Unidos son notablemente más elevados que los salarios referidos en la encuesta española (esta información no está disponible en el trabajo del Reino Unido). Así, en el trabajo de Cawley y Morrisey (2007), las personas que respondieron a la encuesta revelaron unos ingresos medios de 119.499 dólares (93.704 €), ascendiendo esta cifra a 114.573 dólares (89.842 €) en el caso de los economistas académicos y a 128.566 (100.814 $€$ ) en el caso de los economistas no académicos (año base de la valoración, 2005), siendo muy marcadas las diferencias salariales en el sector académico entre categorías profesionales.

Otra información interesante emerge de comparar las respuestas de los ingresos proporcionados por los participantes de nuestro trabajo con los datos de la Encuesta de Estructura Salarial elaborada por el Instituto Nacional de Estadística sobre profesionales con nivel de formación similar. Como ya se indicó en el apartado de resultados, los ingresos declarados por los economistas de la salud son notablemente superiores al de otros profesionales de similar grado de formación. En este sentido, cabe destacar dos elementos que pueden explicar estas diferencias salariales. El primero es la posibilidad de realizar otras tareas fuera de la actividad principal señalada por las personas encuestadas (impartición de cursos de formación, investigación y consultoría remuneradas), así como un elevado número de horas media (47h) de trabajo señaladas por las personas encuestadas. El segundo tiene que ver con aspectos específicos del campo profesional de la economía y gestión de la salud, área donde pueden darse simultáneamente procesos de autoselección en la elección del campo profesional elegido (elevada inversión inicial en formación y adquisición de experiencia antes de recuperar el coste de la misma), junto con barreras a la entrada (derivada de su carácter muy especializado), al tiempo que actúan imperfecciones de información y costes de búsqueda en el mercado de trabajo, unidas a rigideces en el sector educativo (escasez de facultades que imparten en el grado de economía y ADE la asignatura de Economía de la Salud, y nula penetración en la formación de grado en el campo de las ciencias de la salud). En suma, sin atrevernos a afirmar, por falta de los datos necesarios para contrastar la hipótesis, que en el campo profesional de la economía y gestión de la salud se cumpla la «paradoja de Diamond» (los costes de fricción de búsqueda pueden tener un efecto importante sobre los salarios, tanto en términos de elevación como de dispersión) (Diamond, 1971; Montensen y Pissarides, 1999), sí está claro que el comportamiento del mercado español se aleja mucho del esperado de un mercado competitivo, lo cual, en un terreno profesional muy especializado como el que nos ocupa, puede suponer problemas de emparejamiento (demanda superior a la oferta) y diferenciales salariales importantes respecto a otros sectores. 
Una dimensión donde se observaron similitudes claras entre España y Estados Unidos fue el tiempo total dedicado a la semana a las actividades profesionales (alrededor de 47 horas por semana en ambas encuestas). En cambio, los participantes en la encuesta del Reino Unido declararon menos horas semanales dedicadas a actividades profesionales $(40,2)$. La distribución del tiempo según los diferentes entornos profesionales sí muestran importantes diferencias. Por ejemplo, en el caso de los economistas de la salud académicos, se observa mayor tiempo dedicado a la investigación en los Estados Unidos frente a España ( $56 \%$ frente a $45 \%$ ) y menor a la enseñanza (30\% frente a $35 \%$ ). En el caso de los académicos del Reino Unido, se observó que dedicaban una proporción importante de tiempo a la investigación (cerca del 70\%) y una proporción menor a la enseñanza (un 14\%).

En nuestro trabajo, los economistas de la salud entrevistados tienden a publicar sus trabajos en revistas españolas de economía general, revistas internacionales de economía de la salud, revistas nacionales e internacionales sobre salud pública y en revistas de gestión de la salud y de política sanitaria, siendo menos frecuente la publicación de artículos en revistas internacionales de economía general. Estos resultados son coincidentes con estudios anteriores realizados en otros países (Fuchs, 2000; Morrisey y Cawley, 2008). Health Economics y Journal of Health Economics fueron las publicaciones reseñadas como más atractivas para las personas encuestadas en nuestro trabajo. Las revistas de economía del ámbito español no son reconocidas como las más atractivas por las personas encuestadas para publicar sus trabajos dado que la mayoría no tiene factor de impacto, y por lo tanto, su consideración en el curriculum académico no está a la misma altura que una publicación internacional con factor de impacto reconocido. Esta situación es aún más marcada si los encuestados trabajan fuera del ámbito académico. Aunque no existen otros estudios analizando el número de artículos publicados por miembros de otras asociaciones de economistas en España, probablemente el número de publicaciones por investigador sea mayor en el campo de la economía de la salud debido al modelo de investigación colaborativa, más común del ámbito de las ciencias de la salud que de las ciencias sociales.

Una limitación de nuestro estudio es que nuestra tasa de respuesta no supera el $50 \%$ sobre el censo empleado. La tasa de respuesta de nuestro estudio (37\%) es ligeramente superior a la obtenida por Morrisey y Cawley (2008) (32\%), pero inferior al 44\% de Kaambwa y Frew (2013), al 43\% de Resneck y Luft (2004) y al 54\% obtenido por Feldman y Morrisey (1990). Tal y como indican Morrisey y Cawley (2008), una tasa de respuesta relativamente baja plantea el problema en la generalización de los hallazgos a la población analizada. La dirección del sesgo de no-respuesta, si la hay, no es en absoluto evidente. Por un lado, las personas con más ocupaciones y con mayor coste de oportunidad de su tiempo pueden ser menos propensas a responder. Sin embargo, por otra parte, las personas con mayor compromiso e interés profesional en los temas que plantea la encuesta pueden estar más dispuestos a responder. Adicionalmente, una parte de los profesionales que decidieron no participar podrían no considerarse a sí mismos como economistas de la salud y haberse autoexcluido de la respuesta, aun cuando el universo de análisis son las personas con interés en la economía y gestión de la salud (no necesariamente economistas).

Otra cuestión a considerar es que, como hemos señalado, la mayoría de las personas que respondieron a la encuesta son miembros de la Asociación de Economía de la Salud 
(AES). Lógicamente, no podemos afirmar categóricamente que la muestra sea completamente representativa de todos los profesionales con interés en economía de la salud en España. Ello no es posible toda vez que no existe un registro de economistas de la salud o ni siquiera datos accesibles sobre los participantes a las Jornadas de Economía de la Salud. La muestra es autoseleccionada, en el sentido de que hay economistas que trabajan en el ámbito sanitario y de la salud y no están afiliados a AES ni participan o han participado recientemente en sus Jornadas.

Esta limitación no es exclusiva de este trabajo sino compartida con los escasos trabajos similares (Cawley, J. \& Morrisey , 2007; Kaambwa, B. \& Frew , 2013). Sin embargo, las Jornadas de Economía de la Salud son la actividad de referencia en España para la economía de la salud y AES es una asociación que facilita el acceso a un foro de debate y participación a personas con intereses en esta área, a través de una pequeña cuota (6o €), en comparación con los salarios de los profesionales. Por lo tanto, podemos defender con un alto grado de fiabilidad que nuestra encuesta se dirigió al grupo de profesionales con mayor interés aplicado hacia la economía de la salud en España.

Por último, la comparación de las características de las personas con un interés en Economía de la Salud, en España, en los EE.UU. y el Reino Unido muestra resultados interesantes. Futuras investigaciones deberían extender este tipo de estudio a otros países europeos y luego a otros continentes, para así identificar los perfiles de las personas con interés en economía de la salud en un contexto global, y analizar las diferencias y los intereses comunes de los profesionales que trabajan en este área. Asimismo, dentro del medio español, sería interesante repetir el ejercicio para comparar resultados y ampliar alguna de las dimensiones consideradas (por ejemplo, la referida a la investigación y difusión de resultados).

\section{Conclusiones y líneas de avance}

Esta es la primera encuesta de profesionales españoles con interés en economía y gestión de la salud. Nuestros resultados ponen de manifiesto la variedad de profesiones relacionados con esta disciplina, confirmando su carácter multidisciplinar y aportando información sobre aspectos formativos, laborales e investigadores de este colectivo. Esta información no solo puede ser interesante para los propios profesionales con interés en la economía y gestión de la salud sino también para planificar medidas de fomento de los recursos humanos en este ámbito, contando con un escenario de incremento de la demanda de evaluación de políticas de salud. Como futuras líneas de investigación, una segunda encuesta podría ofrecer información sobre el efecto que está teniendo la crisis económica sobre los principales resultados obtenidos. Asimismo, sería interesante ampliar el número de países donde se realizan este tipo de estudios con el fin de mejorar nuestro conocimiento sobre los profesionales que trabajan en el ámbito de la economía y gestión de la salud en todo el mundo.

\section{Agradecimientos}

Agradecemos el apoyo prestado por la Asociación de Economía de la Salud (AES), sin el cual no habría sido posible este trabajo. También queremos agradecer a Enrique Bernal, Carlos Campillo, José Miguel Carrasco, Beatriz González López-Valcárcel, Iván Moreno, 
Salvador Peiró, José Manuel Rodríguez, José Ramón Repullo y Ana Tur por sus útiles comentarios. A Catriona Crossan y Celia Muñoz por tareas de corrección y pruebas. A Michael A Morrisey y John Cawley por su disposición a compartir la encuesta original y sus útiles comentarios. A los revisores de la revista por sus comentarios. Y, por último, a todas las personas que desinteresadamente participaron de manera anónima en la encuesta. Cualquier error es responsabilidad exclusiva de los autores.

\section{Referencias Bibliográficas}

Asociación de Economía de la Salud. (2012). Estatutos (aprobados por l Asamblea General de la Asociación celebrada en Bilbao el 17 de Mayo de 2012). Disponibles en: http://aes.es/admin2/uploads/docs/ESTATUTOS_DE_LA_ASOCIACION_ DE_ECONOMIA_DE_LA_SALUD.pdf (acceso el 19 de octubre de 2014).

Cawley, J. \& Morrisey, M.A. (2007). The earnings of U.S. health economists. Journal of Health Economics, 26, 358-372.

Diamond, P. (1971). A Model of Price Adjustment. Journal of Economic Theory, 3, 156168.

Feldman, R. \& Morrisey, M.A. (1990). Health Economics: a report on the field. Journal of Health Politics Policy and Law, 15(3), 627-646.

Fuchs, V.R. (2000). The future of Health Economics. Journal of Health Economics, 19 (2), 141-158.

Gulacsi, L. (2007). The time for cost-effectiveness in the new European Union member states: the development and role of Health Economics and technology assessment in the mirror of the Hungarian experience. European Journal of Health Economics, $8(2), 83-88$.

Kaambwa, B. \& Frew, E. (2013). Health Economics in the UK: Capacity, constraints and comparisons to US health economists. International Review of Economics Education, 12, 1-11.

Morrisey, M.A. \& Cawley, J. (2008). US Health economists: who we are and what we do. Health Economics, 17, 535-543.

Mortensen, D. y Pissarides, C. (1999). New Developments in Models of Search in the Labor Market, en O. Ashenfelter and D. Card (ed.): Handbook of Labor Economics, volumen 3, Chapter 39, Amsterdam: North-Holland, páginas 2627-2657.

Oliva, J. \& Zozaya, N. (2006). Economía y gestión de la salud. Formación y oportunidades laborales. Gaceta Sanitaria, 20 (Supl 2), 17-28.

Resneck, J. \& Luft, H. (2004). How health policy and health services researchers are compensated: analysis of a Nationwide Salary Survey. Medical Care Research and Review, 61(3), 392-408. 